\title{
EPMA WDS Quality Assurance: Materials and Methods
}

\author{
D. C. Meier, J. M. Davis, R. B. Marinenko, F. N. Meisenkothen, and S. A. Wight
}

Material Measurement Laboratory, National Institute of Standards and Technology, Gaithersburg, MD 20899-8371

The chief product of an analytical laboratory quality assurance (QA) program, ultimately, is confidence-confidence that the analysis of any specimen sent to any laboratory participating in the program will be consistent, correct within tolerance, and interchangeable with equivalent analyses of related specimens performed by any other laboratory in the program. In order to maximize confidence, the QA tests and test materials must be chosen such that they evaluate the broadest possible range of instrument functionality. In the context of electron probe microanalysis (EPMA) wavelength dispersive spectroscopy (WDS), this means testing not only the stability of the electron gun and the function of the photon counters, but the functionality of every component of each wavelength spectrometer mounted to the system. This includes the numerous types of diffracting elements that disperse the X-rays, the mechanical components that flip the spectrometer from one diffractor to another, and the drive belts that scan the diffractor through a region of interest on the Rowland circle. Since these spectrometer components can fail independently of the others and many such failures will not be noticeable in all measurements, a complete QA test must include materials that generate X-ray lines that span the range of any diffractor used in WDS and methods to properly analyze it, thus generating the maximum information on the instrument's function. From this information, instrument performance can be optimized, thereby obtaining maximum analytical confidence.

NIST presently uses a selection of metals, metal oxides, and mineral glasses, specifically K-411 and K-412, as material standards and model unknowns for its weekly QA test measurement on its EPMA WDS instrument. This suite of oxides and glasses is excellent for testing the functionality of a spectrometer featuring a thallium acid phthalate (TAP) crystal diffractor, since the $\mathrm{Mg}, \mathrm{Al}$, and $\mathrm{Si} \mathrm{K}$ lines represent positions on the spectrometer that span nearly $100 \mathrm{~mm}$. As a spectrometer traverses that range, issues such as dragging wires, systematic errors in position, and diffractor defects can be readily identified in a QA test. The $\mathrm{Ca}$ and $\mathrm{Fe}$ that make up the remainder of the test material provide only one element that is measured in a comparatively narrow range on either the pentaerythritol (PET) or the lithium fluoride $(\mathrm{LiF})$ diffractors, respectively, making the results of QA tests for these diffractors less definitive.

An engineered glass (ADM6XXX) has been developed to replace K-412 as the EPMA WDS model unknown. Its composition has been engineered to maximize the specimen's analytical span of the WDS spectrometer for each of three common diffractors (TAP, PET, and LiF). It is being integrated into a comprehensive EPMA WDS QA program that effectively covers most of the periodic table, using a single model unknown and standard specimen block capable of assessing the functionality of every spectrometer on the instrument simultaneously. Under electron beam excitation, this engineered glass emits characteristic X-rays that include first order and second order diffraction lines for two characteristic $\mathrm{K}$ lines on each of the three major diffractor crystals ( $\mathrm{Zn}$ and $\mathrm{Ge}$ on $\mathrm{LiF}, \mathrm{Ca}$ and $\mathrm{Ti}$ on $\mathrm{PET}$, and $\mathrm{Si}$ and $\mathrm{Al}$ on TAP) (Figure 1). This designed material allows for a robust QA to be performed simultaneously on each of these diffractors using a single specimen block, thus providing maximum information for QA evaluation in a minimum amount of instrument time. 
0.55

0.75

0.95

1.15

1.35

1.55

1.75

1.95

2.15

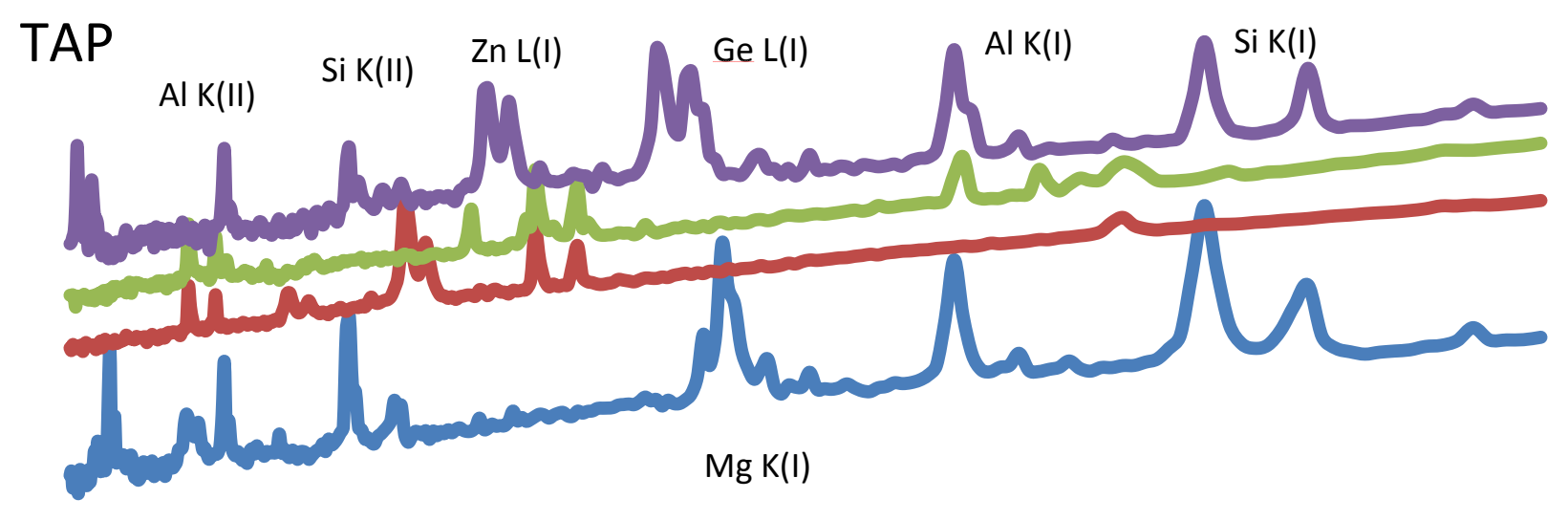

1.5

PET

2.5

3.5

4

4.5

5

5.5

6

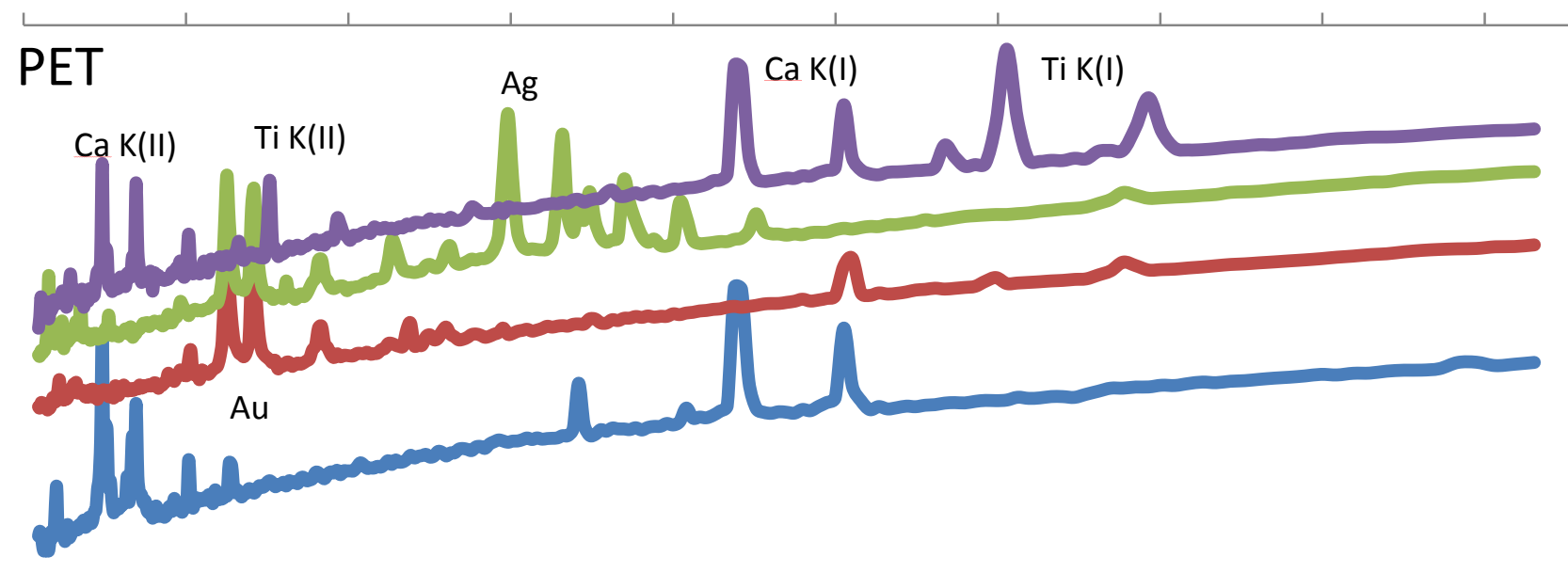

3.2

5.2

7.2

9.2

11.2

13.2

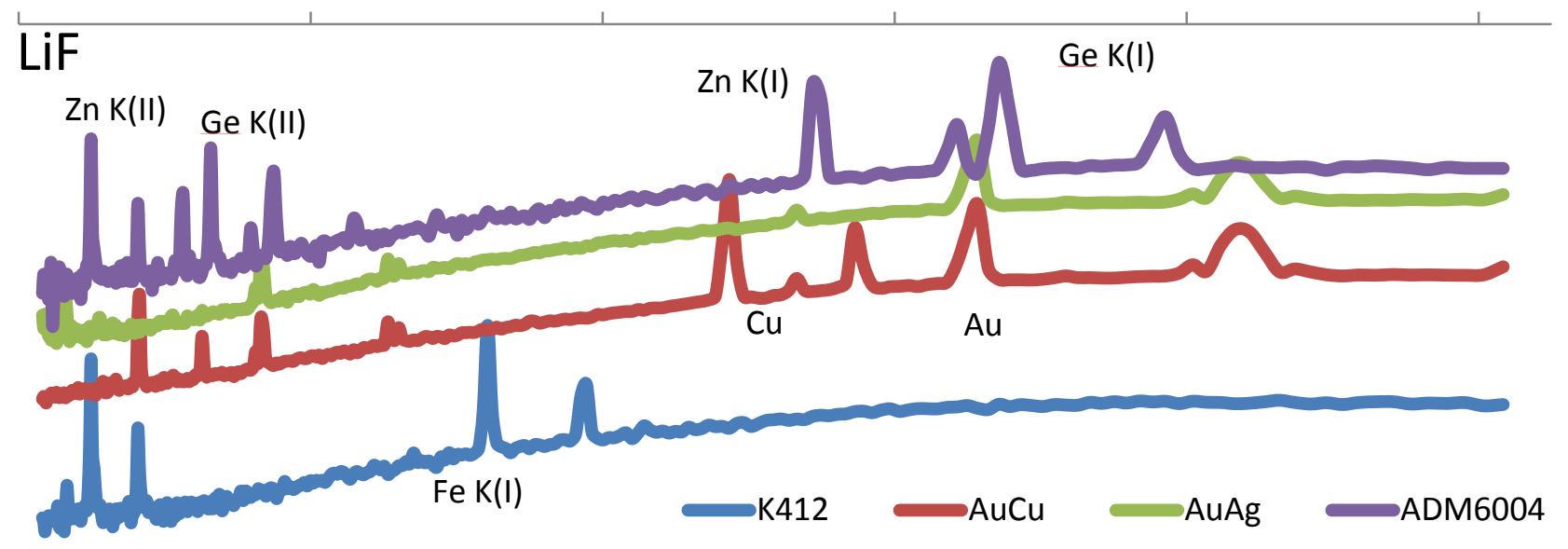

Figure 1. Wavelength spectrometer scans on TAP, PET, and LiF diffractors comparing the spectra of $\mathrm{K}-412$, AuCu and AuAg alloys, and the engineered QA glass (sample ADM6004). 